\title{
Commento al caso clinico di A. Micheloni
}

\author{
Giulio Fabri Poncemi*
}

Per i greci esistevano due parole per descrivere il tempo: Kronos, il tempo oggettivo e lineare, scandito dal susseguirsi dei giorni, delle stagioni e in epoca moderna dall'invenzione degli orologi e Kairos traducibile come 'momento giusto' che si riferisce alla percezione soggettiva di una condizione propizia per una presa di decisione. La collega ha intitolato la sua presentazione 'Mary e l'orologio biologico che fa Tic Tac' che ci rimanda a una dimensione più oggettiva $\mathrm{e}$, non a caso, corporea e biologica del trascorrere del tempo. L'età compresa tra i trenta e trentacinque anni è quella in cui la maggior parte delle donne che lo desiderano costruiscono una famiglia e fanno dei figli, come impunemente la mamma di Mary le rinfaccia. Mary non ha il controllo né su Kronos e né sullo stato di salute del proprio corpo e allora si ribella, distrugge e si autodistrugge. Arriva poi Kairos, il momento giusto, la fibromialgia e l'inizio di un percorso di terapia, una domanda vaga, poco mentalizzata, come accade sempre nei disturbi di somatizzazione.

Nel primo colloquio Mary parla del 'fallimento colossale che è la sua vita'. La terapeuta nel colloquio si sente messa al muro. Riesce però ad osservare il suo controtransfert, a tollerare la confusione, Mary parla del suo dolore a un livello fisico e la terapeuta sente come sente Mary, inizia a sentire dei dolori alla mandibola e al collo. Spesso i pazienti narcisisti inondano la seduta di parole, parlano tanto e velocemente e questo diventa un modo per controllare lo spazio interpersonale lasciando il terapeuta attonito e un po' frastornato: aveva comperato il biglietto per una tragedia (i freudiani ortodossi) o una commedia e si ritrova ad assistere a un monologo. Passiamo quindi dal fallimento colossale della vita di Mary a una sorta di fallimento dell'anamnesi di Mary/paziente. Non sappiamo infatti come la paziente funziona a livello lavorativo (le stesse dinamiche di potere e invidia sono presenti anche sul lavoro? Come si relaziona con i superiori i pari e i sottoposti?) né a livello di amicizie (ha relazioni di lunga durata? ha delle amiche

*Libero professionista, via Trento 49, Parma. E-mail fabri.giulio@gmail.com 
intime con cui parlare quando se la vede male?), in modo da capire se le difficoltà relazionali di Mary siano relegate alla sfera sentimentale o siano pervasive e più in generale se ci sono aree della sua vita in cui ci sono degli investimenti più stabili. Sappiamo poco sia della sua storia, a parte il disturbo alimentare in adolescenza che delle fasi dello sviluppo. Ora, sono sicuro che queste lacune sarebbero facilmente colmabili dalla collega se questa fosse un'intervisione ma preferisco indugiare nell'idea che non sia un caso che queste informazioni manchino nella presentazione e che questo possa far riflettere sul bisogno di Mary di sentire di avere il controllo sull'altro. Il controllo onnipotente, sempre che di questo si tratti, è una difesa la cui funzione è di proiettare la propria aggressività su un oggetto esterno, dai cui attacchi ci si difende tenendolo in scacco. L'aggressività e la distruttività di Mary appaiono ben in evidenza in vari punti del racconto della collega, sia nel presente che nel passato della paziente. Si nota nell'eloquio sarcastico e nell'atteggiamento un po' nichilista con cui la paziente in qualche modo attacca sé stessa sabotando la possibilità di porre pensiero alle proprie emozioni. $\mathrm{La}$ stessa sessualità è infiltrata di aggressività e Mary attira gli uomini nel suo appartamento, li usa e si sente padrona della situazione per poi sentirsi usata e vittima impotente, la dinamica è sempre la stessa solo a ruoli invertiti, questo le impedisce di costruire una relazione stabile: idea di una famiglia che Mary attacca costantemente attaccando in realtà un'immagine ideale di sé che in modo confusivo appartiene talora a lei, talora alla madre.

Più avanti in un passaggio molto indicativo la collega descrive un cambiamento nella terapia. Mary passa da essere spettatrice delle proprie disgrazie ad accogliere l'immagine della terapeuta che la descrive come un felino in caccia. Mary inizia a poter pensare di avere un ruolo più attivo nella costruzione del proprio destino. Sarei curioso di sapere che forma ha preso nella mente di Mary questa presa di coscienza e come si è sviluppata nel transfert. Come la collega ha detto le emozioni sembrano mancare all'appello in questa storia, anche i sistemi motivazionali che ne sono alla base sembrano estremamente inibiti, parlo in particolare dell'aggressività $\mathrm{e}$ del sistema dell'attaccamento e delle emozioni legate alla dipendenza che vi sono legate. La Mary cacciatrice in che modo occupa la relazione terapeutica? L'invidia e la distruttività di Mary sono scisse e agite sul mondo e sul sé, e la terapeuta è salvaguardata e mantenuta in uno stato di idealizzazione? Se la somatizzazione può essere vista come una forma di acting out in cui Mary attacca la terapia attaccando sia la propria capacità di pensare, sia una parte del proprio corpo, è stato possibile far emergere questi sentimenti aggressivi e distruttivi nel transfert?

Concludo ringraziando la collega per aver condiviso questo materiale, estremamente ricco. Rileggendolo più volte colpisce la quantità di spunti e di 'angoli' da cui si può guardare: il tema alessitimico della somatizzazione, il tema del narcisismo, della gestione della rabbia e dell'invidia, più o meno 
inconsce, e quello della malattia fisica. Sarei molto curioso di sapere come proseguirà la terapia di Mary. La stanza in più sarà una dependance del sé grandioso rinforzato dal sacrificio estremo del padre, disposto a rinunciare alla casa costruita con i risparmi di una vita, per rendere felice la figlia o sarà uno spazio abitabile da un sé meno onnipotente e disponibile a un incontro più realistico con l'altro?

Conflitto di interessi: l'autore dichiara che non vi sono potenziali conflitti di interessi.

Approvazione etica e consenso a partecipare: non necessario.

Ricevuto per la pubblicazione: 14 aprile 2021.

Accettato per la pubblicazione: 14 aprile 2021.

${ }^{C}$ Copyright: the Author(s), 2021

Licensee PAGEPress, Italy

Ricerca Psicoanalitica 2021; XXXII:541

doi:10.4081/rp.2021.541

This article is distributed under the terms of the Creative Commons Attribution Noncommercial License (by-nc 4.0) which permits any noncommercial use, distribution, and reproduction in any medium, provided the original author(s) and source are credited. 
